\title{
Effectiveness of corticosteroids
} for post-extubation stridor and extubation failure in pediatric patients: a systematic review
and meta-analysis

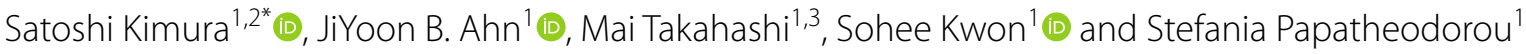

\begin{abstract}
Background: While the results of previous meta-analyses have shown beneficial effects of corticosteroid therapy on post-extubation stridor and extubation failure in adults, these results might not be generalizable to children because of the differences in anatomy and structure. We aimed to determine the benefits of corticosteroids on those outcomes in pediatric populations.

Methods: We searched PubMed, EMBASE, and reference lists of articles from inception until February 2019. Randomized controlled trials and observational studies on the efficacy of systemic corticosteroid administration given prior to elective extubation in mechanically ventilated pediatrics were eligible. Outcomes included post-extubation stridor indicating laryngeal edema and extubation failures.

Results: A total of ten randomized controlled trials with 591 pediatric patients were included: seven of the ten studies for post-extubation stridor/suspected upper airway obstruction and nine of the ten studies for extubation failure. The estimate of pooled odds ratios (ORs) for post-extubation stridor/suspected upper airway obstruction was 0.40 ( $95 \%$ Cl: $0.21-0.79$ ). When analysis was restricted to trials that had explicit data for infants and explicit data for pediatric patients under 5 years old excluding infants, the estimates of pooled ORs were 0.53 ( $95 \%$ Cl: $0.20-1.40$ ) and 0.68 ( $95 \%$ Cl: 0.38-1.22), respectively. For pediatric patients who received corticosteroids, there was a 0.37 -fold lower odds of extubation failure than that in pediatric patients who did not receive corticosteroids $(\mathrm{OR}, 0.37 ; 95 \% \mathrm{Cl}, 0.22-0.61)$. While three observational studies were included in this review, their estimates have a potential for bias and we did not perform a meta-analysis.
\end{abstract}

Conclusions: Despite a relatively small sample size in each randomized controlled trial and wide ranges of ages and steroid administration regimens, our results suggest that the use of corticosteroids for prevention of post-extubation stridor and extubation failure could be considered to be acceptable in pediatric patients.

Keywords: Mechanical ventilation, Breathing, Laryngeal edema, Respiratory sounds, Glucocorticoids, Pediatrics

\footnotetext{
*Correspondence: kimsato1034@hotmail.co.jp

${ }^{2}$ Department of Pediatric Intensive Care Unit, The Royal Children's

Hospital, 50 Flemington Rd, Parkville, VIC 3052, Australia

Full list of author information is available at the end of the article
}

\section{Background}

Laryngeal edema can cause acute upper airway obstruction and is one of the life-threatening sequelae associated with mechanical ventilation under endotracheal intubation. Mechanical pressure or irritation from the endotracheal tube is associated with the potential development of laryngeal edema, resulting in stridor upon extubation $[1,2]$. The incidence of post-extubation laryngeal edema 
in critical care patients, characterized by stridor and/or respiratory distress, ranges from $4-37 \%$ depending on the population being investigated [3-8]. Post-extubation laryngeal edema increases the probability of reintubation, leading to nosocomial pneumonia, a prolonged duration of endotracheal intubation, a longer duration of stay in the intensive care unit (ICU), and high mortality $[9,10]$.

Post-extubation edema or stridor following endotracheal intubation is more commonly symptomatic in children than in adults due to differences in airway anatomy $[1,7,11]$. Compared with adults, the submucosal connective tissue in the subglottic area is looser in children, allowing fluid to accumulate [1]. In addition, the less expandable cricoid cartilage ring around the trachea and the smaller size of the larynx in children make the effect of reduction in airway diameter, even a small amount of edema, more likely to result in infringement of the crosssectional area of the airway and large airway resistance, causing more severe airflow limitation $[1,2]$.

There have been many studies on the effectiveness of intravenous corticosteroids for preventing or treating this life-threatening complication. Theoretically, corticosteroids may be effective due to their anti-inflammatory effects for reducing laryngeal infiltration of inflammatory cells and for reducing edema $[2,12,13]$. Recently, meta-analyses have shown beneficial results of corticosteroid therapy for prevention of post-extubation stridor and reintubation in adults $[2,14,15]$. However, evidence for the benefits of the administration of steroids in adults may not be generalizable to neonates or children because of the differences in anatomy in pediatric patients. In this meta-analysis, we aimed to determine the effects of corticosteroids, comparing to no corticosteroid use, on laryngeal edema possibly resulting in post-extubation stridor or other findings indicating laryngeal edema and extubation failure in a pediatric population (neonates, infants, children or adolescents) and to determine potential adverse effects of corticosteroids in this population.

\section{Methods}

\section{Literature search}

We searched PubMed and EMBASE from inception until February 2019 by using MESH terms and synonyms of "laryngeal edema", "extubation" and "corticosteroids". We also reviewed the references of retrieved articles for potentially eligible trials. The electronic search strategies are shown in Appendix 1.

\section{Eligibility criteria}

We followed the Preferred Reporting Items for Systematic Reviews and Meta-Analyses (PRISMA) statement to select relevant studies for our research [16].
We included randomized controlled trials (RCTs) and observational studies on the efficacy of systemic corticosteroid administration given prior to elective extubation in mechanically ventilated pediatrics (neonates, infants, children or adolescents). We excluded trials in which corticosteroids were administered only after extubation, trials in which nebulized corticosteroids were used, trials in which a comparison was made with other interventions, trials that included patients with potentially steroid-effective diseases such as croup, asthma, and respiratory syncytial virus infection, and trials for which reports were not written in English.

\section{Data collection}

We used the online Covidence software [17] for screening, selection, and data extraction. All records were independently evaluated by two members of the authors' team (SK, JA) through title and abstract screening. Disagreements regarding title and abstract eligibility were resolved by a discussion between the two members as well as a third author (MT). Full text screening of selected papers was conducted by two members of the authors' team (SK, JA). Disagreements regarding record eligibility were resolved by a discussion between the two authors and a third author (MT) cast a final vote if a consensus was not reached. All studies meeting the inclusion criteria were retained in the analysis. Access to all full texts could be obtained without having to contact corresponding authors. Two authors also independently extracted the following data from each study included in our analysis: first author, publication year, location, study setting, sample size, gender, age, regimen of corticosteroid administration, observational duration, and frequencies of manifestations related to laryngeal edema such as stridor and reintubation. When available, we recorded data for adverse events such as hyperglycemia, hypertension, infection, and gastrointestinal bleeding. Corticosteroid doses were standardized to hydrocortisone equivalents, according to the relative anti-inflammatory potency of each drug: $25 \mathrm{mg}$ hydrocortisone being equivalent to $5 \mathrm{mg}$ methylprednisolone or $1 \mathrm{mg}$ dexamethasone [14].

We assessed the risk of bias using the Cochrane risk of bias assessment tool for RCTs [18]. We also assessed the risk of bias using the Newcastle-Ottawa Scale for nonrandomized studies [19], considering studies achieving 6 points or more as high quality [20]. In addition to the scale, we emphasized comparability between groups because of the importance for validity of observational studies. Any conflicts or disagreements in the whole process were solved by discussion and consensus. 


\section{Outcomes and data analysis}

The primary outcome was post-extubation stridor/suspected upper airway obstruction indicating laryngeal edema related to endotracheal intubation. Secondary outcomes were extubation failures defined as reintubation or use of non-invasive ventilation within $72 \mathrm{~h}$ after extubation and adverse effects related to corticosteroids. Binary outcomes were pooled by using a random-effect model for incidence of stridor/suspected upper airway obstruction in order to take clinical heterogeneity of the included populations and other sources of variation between studies into account. The DerSimonian and Laird random-effects model [21] was applied for the primary outcome with the odds ratio (OR) and $95 \%$ confidence interval (CI). A fixed-effect model with the Peto method [22] was used for the incidence of extubation failure to address rare events.

We evaluated heterogeneity between studies with the Cochrane $X^{2}$ test [23] and the $I^{2}$ statistic with an $I^{2}$ value higher than $50 \%$ indicating at least moderate heterogeneity [24]. Publication bias was assessed by visual inspection of the funnel plot and with the Egger test [25]. A sensitivity analysis for post-extubation stridor/ suspected upper airway obstruction was performed by excluding trials in which stridor was used as one of the scores for upper airway obstruction, but other scores in the scoring system might be related to conditions other than laryngeal edema. Trials that used outcomes other than reintubation and additional airway manipulation as "extubation failure" and trials that included neonates with extremely low birth weights and in which corticosteroids were administered for more than 10 days for other purposes were excluded in a sensitivity analysis for extubation failure. Meta-regression was conducted for the associations of outcomes with cumulative dose of corticosteroid equivalent to hydrocortisone, timing of first corticosteroid treatment before extubation, and age. The statistical significance was set at $p<0.05$ two-sided. All statistical analyses were performed using R 3.6.0 (R foundation for Statistical Computing, Vienna, Austria).

\section{Results}

\section{Overviews of randomized controlled trials and risk of bias} assessment

The search retrieved a total of 517 references. After application of the inclusion and exclusion criteria, $10 \mathrm{RCTs}$ were included in this review. A PRISMA flowchart of this process is shown in Fig. 1. Details of the included RCTs are shown in Additional file 1: Table S1. There was one study restricted to neonates [26] and three studies included only infants [27-29], but the rest of the studies included pediatric patients with a wide range of ages. One study included participants with known laryngeal edema [30], six studies included patients at high risk for laryngeal edema such as patients with prolonged intubation and mechanical ventilation $[11,27,28,31-33]$, and

517 references retrieved from all searches

455 references excluded after screening abstracts

62 full text articles retrieved for full review

49 articles excluded for the following reasons

- No detailed data about stridor and/or re-intubation $(n=5)$

- No steroid administration before extubation $(n=4)$

- No valid comparison of steroid with placebo in RCT $(n=2)$

- Not in English ( $\mathrm{n}=7$ )

- No placebo in the control in RCT $(n=2)$

- Poster or letter $(n=5)$

- Review $(n=4)$

- Systematic review $(\mathrm{n}=6)$

- Wrong route of steroid administration $(n=1)$

- Wrong study population $(\mathrm{n}=7)$

- Duplicate articles $(n=6)$

10 randomized controlled trials and 3 non-randomized controlled trials included in meta-analysis

Fig. 1 Study selection workflow 
three studies included participants to whom steroids were given for another reason such as expectation of improved pulmonary function [26, 29, 34]. Dexamethasone was used in nine studies [11, 26-33] and methylprednisolone was used in one study [34] for intervention. There was a range of different regimens of dosing, frequency, and timing for administration of corticosteroids among the trials (Additional file 1: Table S1).

Eight (80\%) out of ten trials and four (40\%) out of ten trials had a low risk of bias in random sequence generation and allocation concealment, respectively. Reports of two trials explicitly mentioned both blinding of participants and personnel and blinding of outcome assessors. Eight trials showed a low risk of bias in incomplete outcome data. A summary of the risk of bias assessment for the RCTs is shown in Fig. 2.

\section{Overview of observational studies and risk of bias assessment}

After application of the inclusion and exclusion criteria, three observational studies were included in this review [35-37]. Details of the observational studies are shown in Additional file 1: Table S2. One of the studies was a prospective cohort study [37] and there were two retrospective cohort studies $[35,36]$.

All of the observational studies showed a NewcastleOttawa Quality score of 6, which is classified as "high quality" (Additional file 1: Table S3). However, in those studies, the relationship between corticosteroids and post-extubation stridor was estimated by just comparing two groups without adjustment for confounders. Furthermore, as shown in Additional file 1: Table S2, only adjustment for age was made in one study and there was no adjustment for any potential confounders in the other two studies. Given that the groups were not comparable in their baseline characteristics, their estimates have a potential for bias. Therefore, we did not perform a meta-analysis.

\section{Post-extubation stridor/suspected upper airway obstruction}

Reports for seven of the 10 RCTs, including 453 patients, showed the incidence of stridor/suspected upper airway obstruction after extubation $[11,27,28,31-34]$. The estimate of pooled ORs for post-extubation stridor was 0.40 (95\% CI: $0.21-0.79, I^{2}=47 \% ; p=0.08$ ), indicating significantly lower odds of stridor with the use of corticosteroids (Fig. 3). Publication bias was not assessed by the funnel plot and the Egger test because of the small number of studies. In sensitivity analysis, including six trials that used stridor as a main assessment $[11,27,28$, 32-34], the estimate of pooled ORs for post-extubation stridor was 0.36 (95\% CI: $0.17-0.74, I^{2}=51 \% ; p=0.07$ ) (Additional file 2 Figure S1A).

When analysis was restricted to trials with data for infants $[27,28,31,32]$, the estimate of pooled ORs was 0.53 (95\% CI: $0.20-1.40, I^{2}=41 \% ; p=0.16$ ) (Additional file 2: Figure $\mathrm{S1B}$ ). When analysis was restricted to trials with data for pediatrics under five years old, not including infants [31, 32], the estimate of pooled ORs was 0.68 (95\% CI: $0.38-1.22, I^{2}=0 \% ; p=0.75$ ) (Additional file 2: Figure $\mathrm{S} 1 \mathrm{C}$ ). It was not feasible to conduct other agesubgroup analyses because most of the studies included a wide range of age groups and did not have accurate data for the incidence based on age categories.

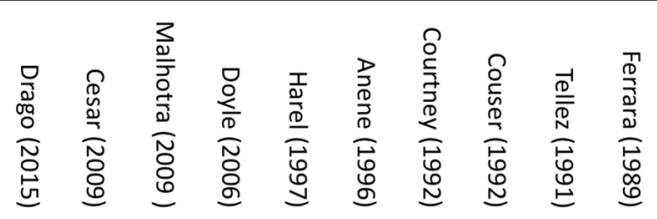

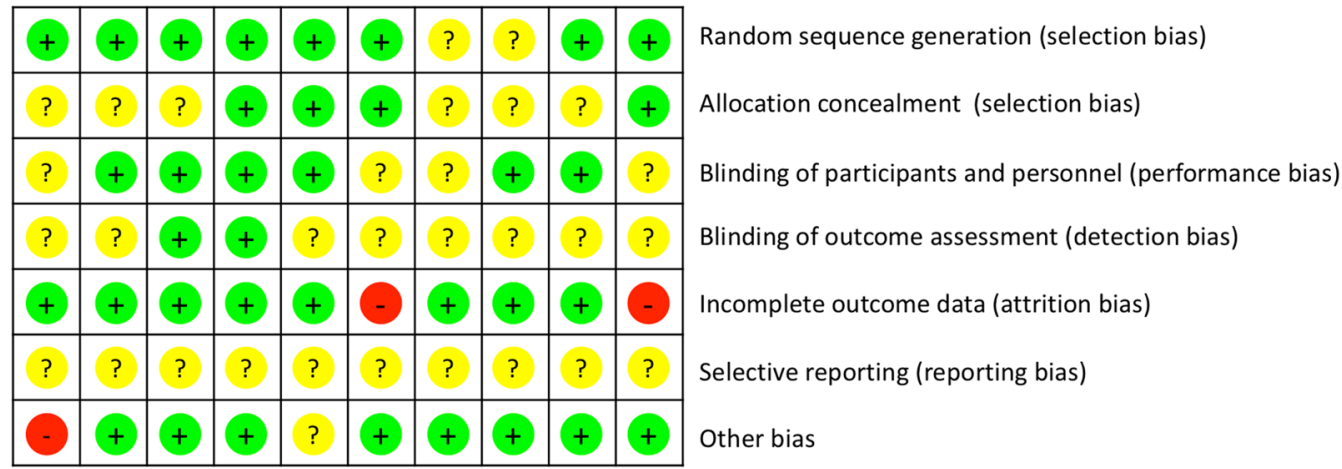

Fig. 2 Risk of bias summary of randomized controlled trials 




\section{Extubation failure}

Reports for nine of the $10 \mathrm{RCTs}$, including 553 patients, showed the incidence of extubation failure [11, 26-33]. Pediatric patients who received corticosteroids had a 0.37 -fold lower odds of extubation failure than did those who did not receive corticosteroids (OR, 0.37; 95\% CI, $0.22-0.61 ; I^{2}=59 \% ; p=0.02$ ) (Fig. 4). Publication bias was not assessed by the funnel plot and the Egger test because of the small number of studies. In sensitivity analysis, excluding one trial that included patients with acute respiratory distress syndrome and in which death and withdrawal from the study, as well as reintubation, were defined as extubation failure [26], the estimate of pooled ORs was 0.45 (95\% CI: $0.26-0.80, I^{2}=52 \%$; $p=0.05)$.

When restricting analysis to trials with data in infants in the sensitivity analysis [27-29], the estimate of pooled ORs for extubation failure was 0.16 (95\% CI: $0.03-0.92, I^{2}=0 \% ; p=0.52$ ) (Additional file 3: Figure S2). Other age-subgroup analyses could not be conducted because patients with a wide range of ages were included in each study.

\section{Adverse effects}

Potential adverse effects of corticosteroids were infrequently assessed in the RCTs. Among them, hyperglycemia was the most frequently assessed potential adverse effect of corticosteroids, followed by gastrointestinal bleeding, infection, hypertension, and glycosuria (Table 1). Because of the small sample size and infrequent reporting of adverse effects of corticosteroids, we could not robustly assess the adverse effects of corticosteroids for pediatric patients.




Table 1 Adverse events reported as potentially attributable to corticosteroids

\begin{tabular}{|c|c|c|c|}
\hline Adverse event & Author & Control & Steroids \\
\hline Gastrointestinal bleeding & Anene et al. [33] & $0 / 32$ & $1 / 32$ \\
\hline \multirow[t]{4}{*}{ Hyperglycemia } & Anene et al. [33] & $0 / 32$ & $0 / 32$ \\
\hline & Drago et al. [34] & $8 / 18$ & $10 / 17$ \\
\hline & Doyle et al. [26] & No significant differences in blood glucose concentration & \\
\hline & Courtney et al. [29] & Mean serum glucose 87 mg/dl & $\begin{array}{l}\text { Mean serum } \\
\text { glucose } \\
101 \mathrm{mg} / \mathrm{dl}\end{array}$ \\
\hline \multirow[t]{2}{*}{ Infection } & Drago et al. [34] & $5 / 18$ & $1 / 17$ \\
\hline & Courtney et al. [29] & $1 / 23$ & $1 / 19$ \\
\hline \multirow[t]{2}{*}{ Hypertension } & Anene et al. [33] & $1 / 32$ & $1 / 32$ \\
\hline & Doyle et al. [26] & No significant differences in blood pressure & \\
\hline Glucosuria & Couser et al. [27] & $0 / 23$ & $7 / 27$ \\
\hline
\end{tabular}

\section{Meta-regression analysis}

In meta-regression analysis for the RCTs that provided enough evidence, [11, 26-34], we assessed whether there were a linear relationships between the effect size and covariates including cumulative corticosteroid dose, time from the first administration to extubation, and mean (or median) age. As shown in Additional file 1: Table S4, each coefficient of those covariates did not have statistical significance.

\section{Discussion}

The effects of corticosteroids on laryngeal edema possibly resulting in stridor after and on extubation failure in pediatric patients were assessed in this study. Although there were wide ranges of corticosteroid regimens, cumulative doses, and frequencies and durations of administration among those trials, our meta-analyses demonstrated that corticosteroids significantly reduce the rates of post-extubation stridor/suspected upper airway obstruction and extubation failure when used in pediatric patients with or at risk of laryngeal edema. Although only a limited number of subgroup analyses for different age categories could be performed because each trial included children with various ages, the analyses showed neither a significant difference in odds of stridor for pediatric patients under five years old, not including infants, nor a significant difference in odds of extubation failure for infants.

In 2009, McCaffey et al. conducted the latest systematic review and meta-analysis, including children and adults, and concluded that corticosteroid administration is effective for preventing laryngeal edema (OR, 0.36; 95\% CI, 0.27-0.49) and for reducing the incidence of extubation failure (OR, 0.56; 95\% CI, 0.41-0.77) in critically ill patients of all ages [15]. However, there are some concerns about their conclusion. First, although the authors included three papers for neonates and four papers for pediatrics in their analyses, they combined those data with data for adults and analyzed the data together. Since the airway anatomy and structure are different for different ages, it may be inappropriate to analyze those data together. Second, they included a study conducted by Tibballs et al., which included children with croup [38]. Since croup is one of the diseases in which corticosteroids are effective, those patients should be excluded from a meta-analysis to assess the effect of corticosteroids on laryngeal edema. In the same year, a Cochrane Systematic Review [2] evaluated the effects of corticosteroids for these age groups separately. Two trials in neonates $(n=109)$ and two trials in children $(n=216)$ were included. The results of their meta-analyses showed that there was no statistically significant reduction in the rate of reintubation among neonates (risk ratio (RR), 0.10; 95\% CI, 0.01-1.68) or among children (RR, 0.49; 95\% CI, 0.01-19.65) and that there was no statistically significant reduction in the incidence of post-extubation stridor among neonates (RR, 0.42; 95\% CI, 0.07-2.32). They only found a significant reduction in the incidence of postextubation stridor among children with heterogeneity (RR, 0.53; 95\% CI, 0.28-0.97; $I^{2}=53 \%$ ). Based on these results, they concluded that the use of corticosteroids to prevent stridor after extubation has not been proven to be effective for neonates or children [2] and there has not been a consensus regarding the recommendation of corticosteroids for preventing post-extubation stridor in pediatric patients [39].

In order to reassess the effectiveness of corticosteroids for prevention of laryngeal edema in pediatric patients at this time, about 10 years after those meta-analyses were conducted [2, 15], we included three additional trials for stridor/suspected upper airway obstruction [11, 31, 34] ( $n=453$ in total, compared to 325 in the previous 
meta-analysis [2]; 39.4\% increase in sample size). After excluding one study in which not only stridor, but also other elements for the scoring assessment that might not be related to upper airway obstruction were used [31], we obtained consistent results regarding the significant effect of corticosteroids. We also included five additional trials for extubation failure [11, 26, 29-31] $(n=556$ in total, compared to 325 in the previous meta-analysis [2]; $71.1 \%$ increase in sample size). Trials for patients with respiratory distress disease were excluded in sensitivity analysis because corticosteroids might have an impact on pulmonary function and the incidence of reintubation, but there was still a significant effect on incidence of extubation failure. Our results suggest that the use of corticosteroids for prevention of post-extubation stridor and extubation failure should be considered to be acceptable in pediatric patients until clear evidence is provided by large-scale and appropriately conducted RCTs.

One of the main purposes of this review was to assess the effects of corticosteroids separately by age groups. Due to the lack of sufficient data and the wide range of age groups in each study, however, we could conduct only a limited number of subgroup analyses for infants and children under 5 years old. In these subgroup analyses, we did not find significant effect of corticosteroids on either outcome except for the effect of corticosteroids for extubation failure in infants. Because of the small sample size and/or high heterogeneity, however, it is difficult to reject the effect of corticosteroids for the other outcome or in different age group. In addition, there was also heterogeneity in the indication of corticosteroids among the selected population. Corticosteroids were given for patients with known laryngeal edema, patients at high risk for laryngeal edema, or another reason. Since it is difficult to conduct subgroup analyses considering both indication of corticosteroids and age, it is still unclear which population should receive corticosteroids before extubation.

Meta-regression analyses for stridor/suspected upper airway obstruction showed that cumulative dose of corticosteroids and time from the first dosing to extubation have negative coefficients in each model. Although they did not show statistical significance, the trends of more effectiveness for a larger amount of cumulative corticosteroid dose and for longer duration between the first dosing and planned extubation are consistent with results from a Cochrane Systematic Review conducted by Khemani et al., showing that corticosteroids are more effective with multiple doses administered $12-24 \mathrm{~h}$ prior to extubation [2]. A positive coefficient of age in metaregressions could be interpreted as beneficial effects of corticosteroids for younger patients, but it was also not statistically significant.
Although our study provides the latest insight regarding this topic using rigorous methodology, this study also has some limitations. First, the sample sizes of the primary studies were relatively small and it is likely that some data for outcomes were not reported. However, exchangeability between the compared groups was demonstrated in the primary studies, and we can therefore assume that randomization was effective. Although the effects of corticosteroids on stridor and reintubation were statistically significant, sample sizes might have been too small to detect significant differences in the effects for different ages, different cumulative amounts of corticosteroids, and different durations between the first administration and extubation in the meta-regression analysis. In addition, it was not possible to conduct some subgroup analyses based on age groups in our study, and some analyses restricted to certain age groups failed to show significant effects of corticosteroids on both outcomes, possibly because of the small sample size. Second, the studies included in our meta-analysis differed in terms of age and corticosteroid regimens as shown in Additional file 1: Table S1 and there was statistical heterogeneity with relatively high $I^{2}$ and $\tau^{2}$. Although the same intravenous corticosteroid was used in all but one of the studies, heterogeneity of age and detailed corticosteroid regimen makes it difficult to assess the dose-response effects of those medications based on the available data. In addition, possible difference of the type of tracheal tube, cuffed tube or uncuffed tube, in each patient and in each study could have different impact on the incidence and the severity of laryngeal edema, potentially contributing to the heterogeneity. We were not able to test for publication bias in our analysis because less than 10 studies were available, therefore the tests would be underpowered. Finally, because of the small sample sizes and infrequent reporting of adverse effects of corticosteroids, we could not robustly assess the adverse effects of corticosteroids for those pediatric patients.

\section{Conclusions}

Our meta-analysis showed that the use of corticosteroids is associated with significant reductions in post-extubation stridor and extubation failure in pediatric patients. These findings need careful interpretations because of a relatively small number of sample size in each RCT and a wide range of ages and corticosteroid administration regimens. Larger and properly conducted RCTs are needed to verify our results and provide adequate evidence of the side-effects.

\section{Supplementary information}

Supplementary information accompanies this paper at https://doi. org/10.1186/s13613-020-00773-6. 


\section{Abbreviations}

Cl: Confidence interval; ICU: Intensive Care Unit; OR: Odds ratio; PRISMA: Preferred Reporting Items for Systematic Reviews and Meta-Analyses; RCT: Randomized controlled trial; RR: Risk ratio.

\section{Acknowledgements}

Not applicable.

\section{Authors' contributions}

SK had full access to all the data in the study and takes responsibility for the integrity of the data and the accuracy of the data analysis. JA, MT, SK, and SP contributed substantially to the study design, data analysis and interpretation, and the writing of the manuscript. All authors read and approved the final manuscript.

\section{Funding}

The authors declare that they have no funding.

\section{Availability of data and materials}

The datasets used and/or analysed during the current study are available from the corresponding author on reasonable request.

Ethics approval and consent to participate

Not applicable.

\section{Consent for publication}

Not applicable.

\section{Competing interests}

The authors declare that they have no competing interests.

\section{Author details}

${ }^{1}$ Department of Epidemiology, Harvard T.H. Chan School of Public Health, 677 Huntington Avenue, Boston, MA 02115, USA. ${ }^{2}$ Department of Pediatric Intensive Care Unit, The Royal Children's Hospital, 50 Flemington Rd, Parkville, VIC 3052, Australia. ${ }^{3}$ Department of Internal Medicine, Mount Sinai Beth Israel, 317 E 17th St, New York, NY 10003, USA.

Additional file 1: Table S1. Summary of the characteristics of randomized controlled trials for assessment of post-extubation stridor and/or extubation failure. Table S2. Summary of the characteristics of observational studies for assessment of post-extubation stridor and/or extubation failure. Table S3. Quality assessment of observational studies. Table S4. Meta-regression analysis of post-extubation stridor and reintubation.

Additional file 2: Figure S1. (A) Result of meta-analysis for effects of corticosteroids on incidence of stridor in randomized controlled trials. Results of meta-analysis for effects of corticosteroids on incidence of stridor/ suspected upper airway obstruction among (B) infants and (C) pediatrics under five years old, not including infants in randomized controlled trials. An odds ratio (OR) less than 1 favors corticosteroid administration.

Additional file 3: Figure S2. Results of meta-analysis for effects of corticosteroids on incidence of extubation failure among infants in randomized controlled trials. An odds ratio (OR) less than 1 favors corticosteroid administration.

Received: 29 August 2020 Accepted: 7 November 2020

Published online: 18 November 2020

\section{References}

1. Roberts RJ, Welch SM, Devlin JW. Corticosteroids for prevention of postextubation laryngeal edema in adults. Ann Pharmacother. 2008:42(5):686-91.

2. Khemani RG, Randolph A, Markovitz B. Corticosteroids for the prevention and treatment of post-extubation stridor in neonates, children and adults. Cochrane Datab Syst Rev. 2009;3:Cd001000.
3. Jaber S, Chanques G, Matecki S, Ramonatxo M, Vergne C, Souche B, et al. Post-extubation stridor in intensive care unit patients. Risk factors evaluation and importance of the cuff-leak test. Intensive Care Med. 2003;29(1):69-74.

4. Kriner EJ, Shafazand S, Colice GL. The endotracheal tube cuffleak test as a predictor for postextubation stridor. Respir Care. 2005;50(12):1632-8.

5. Cheng KC, Chen CM, Tan CK, Chen HM, Lu CL, Zhang H. Methylprednisolone reduces the rates of postextubation stridor and reintubation associated with attenuated cytokine responses in critically ill patients. Minerva Anestesiol. 2011;77(5):503-9.

6. Ho LI, Harn HJ, Lien TC, Hu PY, Wang JH. Postextubation laryngeal edema in adults. Risk factor evaluation and prevention by hydrocortisone. Intensive Care Med. 1996;22(9):933-6.

7. Kemper KJ, Benson MS, Bishop MJ. Predictors of postextubation stridor in pediatric trauma patients. Crit Care Med. 1991;19(3):352-5.

8. Darmon JY, Rauss A, Dreyfuss D, Bleichner G, Elkharrat D, Schlemmer $B$, et al. Evaluation of risk factors for laryngeal edema after tracheal extubation in adults and its prevention by dexamethasone. A placebo-controlled, double-blind, multicenter study. Anesthesiology. 1992;77(2):245-51.

9. Torres A, Gatell JM, Aznar E, el-Ebiary M, Puig de la Bellacasa J, Gonzalez $J$, et al. Re-intubation increases the risk of nosocomial pneumonia in patients needing mechanical ventilation. Am J Respir Crit Care Med. 1995;152(1):137-41.

10. Esteban A, Alia I, Tobin MJ, Gil A, Gordo F, Vallverdu I, et al. Effect of spontaneous breathing trial duration on outcome of attempts to discontinue mechanical ventilation. Spanish Lung Failure Collaborative Group. Am J Respir Crit Care Med. 1999;159(2):512-8.

11. Malhotra D, Gurcoo S, Qazi S, Gupta S. Randomized comparative efficacy of dexamethasone to prevent postextubation upper airway complications in children and adults in ICU. Ind J Anaesth. 2009:53(4):442-9.

12. Way WL, Sooy FA. Histologic changes produced by endotracheal intubation. Ann Otol Rhinol Laryngol. 1965;74(3):799-812.

13. Rhen T, Cidlowski JA. Antiinflammatory action of glucocorticoids-new mechanisms for old drugs. N Engl J Med. 2005;353(16):1711-23.

14. Kuriyama A, Umakoshi N, Sun R. Prophylactic corticosteroids for prevention of postextubation stridor and reintubation in adults: a systematic review and meta-analysis. Chest. 2017;151(5):1002-10.

15. McCaffrey J, Farrell C, Whiting P, Dan A, Bagshaw SM, Delaney AP. Corticosteroids to prevent extubation failure: a systematic review and meta-analysis. Intensive Care Med. 2009;35(6):977-86.

16. Moher D, Liberati A, Tetzlaff J, Altman DG, Group P. Preferred reporting items for systematic reviews and meta-analyses: the PRISMA statement. PLoS Med. 2009;6(7):e1000097.

17. Covidence systematic review software, Veritas Health Innovation, Melbourne, Australia. www.covidence.org.

18. Higgins JP, Altman DG, Gotzsche PC, Juni P, Moher D, Oxman AD, et al. The Cochrane Collaboration's tool for assessing risk of bias in randomised trials. BMJ. 2011:343:d5928.

19. https://www.ohri.ca/programs/clinical_epidemiology/oxford.asp.

20. Glenn AJ, Viguiliouk E, Seider M, Boucher BA, Khan TA, Blanco Mejia S, et al. Relation of vegetarian dietary patterns with major cardiovascular outcomes: a systematic review and meta-analysis of prospective cohort studies. Front Nutr. 2019;6:80

21. DerSimonian R, Laird N. Meta-analysis in clinical trials. Control Clin Trials. 1986;7(3):177-88

22. Yusuf S, Peto R, Lewis J, Collins R, Sleight P. Beta blockade during and after myocardial infarction: an overview of the randomized trials. Prog Cardiovasc Dis. 1985;27(5):335-71

23. Cochran WG. The comparison of percentages in matched samples. Biometrika. 1950;37(3-4):256-66

24. Higgins JP, Thompson SG, Deeks JJ, Altman DG. Measuring inconsistency in meta-analyses. BMJ. 2003;327(7414):557-60.

25. Egger M, Davey Smith G, Schneider M, Minder C. Bias in meta-analysis detected by a simple, graphical test. BMJ. 1997;315(7109):629-34.

26. Doyle LW, Davis PG, Morley CJ, McPhee A, Carlin JB. Low-dose dexamethasone facilitates extubation among chronically ventilator-dependent infants: a multicenter, international, randomized, controlled trial. Pediatrics. 2006:117(1):75-83. 
27. Couser RJ, Ferrara TB, Falde B, Johnson K, Schilling CG, Hoekstra RE. Effectiveness of dexamethasone in preventing extubation failure in preterm infants at increased risk for airway edema. J Pediatr. 1992;121(4):591-6.

28. Ferrara TB, Georgieff MK, Ebert J, Fisher JB. Routine use of dexamethasone for the prevention of postextubation respiratory distress. J Perinatol. 1989;9(3):287-90.

29. Courtney SE, Weber KR, Siervogel RM, Spohn WA, Guo S, Malin SW, et al. Effects of dexamethasone on pulmonary function following extubation. J Perinatol. 1992:12(3):246-51.

30. Harel Y, Vardi A, Quigley R, Brink LW, Manning SC, Carmody TJ, et al. Extubation failure due to post-extubation stridor is better correlated with neurologic impairment than with upper airway lesions in critically ill pediatric patients. Int J Pediatr Otorhinolaryngol. 1997;39(2):147-58.

31. Cesar RG, de Carvalho WB. L-Epinephrine and dexamethasone in postextubation airway obstruction: a prospective, randomized, double-blind placebo-controlled study. Int J Pediatr Otorhinolaryngol. 2009;73(12):1639-43.

32. Tellez DW, Galvis AG, Storgion SA, Amer HN, Hoseyni M, Deakers TW. Dexamethasone in the prevention of postextubation stridor in children. J Pediatr. 1991:118(2):289-94.

33. Anene O, Meert KL, Uy H, Simpson P, Sarnaik AP. Dexamethasone for the prevention of postextubation airway obstruction: a prospective randomized, double-blind, placebo-controlled trial. Crit Care Med. 1996:24(10):1666-9
34. Drago BB, Kimura D, Rovnaghi CR, Schwingshackl A, Rayburn M, Meduri GU, et al. Double-blind, placebo-controlled pilot randomized trial of methylprednisolone infusion in pediatric acute respiratory distress syndrome. Pediatr Crit Care Med. 2015;16(3):e74-81.

35. Lukkassen IM, Hassing MB, Markhorst DG. Dexamethasone reduces reintubation rate due to postextubation stridor in a high-risk paediatric population. Acta Paediatr. 2006;95(1):74-6.

36. Saleem AF, Bano S, Haque A. Does prophylactic use of dexamethasone have a role in reducing post extubation stridor and reintubation in children? Indian J Pediatr. 2009;76(5):555-7.

37. Laham JL, Breheny PJ, Rush A. Do clinical parameters predict first planned extubation outcome in the pediatric intensive care unit? J Intensive Care Med. 2015;30(2):89-96.

38. Tibballs J, Shann FA, Landau LI. Placebo-controlled trial of prednisolone in children intubated for croup. Lancet. 1992;340(8822):745-8.

39. Khemani RG, Randolph A, Markovitz B. Steroids for post extubation stridor: pediatric evidence is still inconclusive. Intensive Care Med. 2010;36(7):1276-7.

\section{Publisher's Note}

Springer Nature remains neutral with regard to jurisdictional claims in published maps and institutional affiliations.

\section{Submit your manuscript to a SpringerOpen ${ }^{\circ}$ journal and benefit from:}

- Convenient online submission

- Rigorous peer review

- Open access: articles freely available online

- High visibility within the field

Retaining the copyright to your article

Submit your next manuscript at $\boldsymbol{\nabla}$ springeropen.com 